\title{
Research on the Structure of Principal's Moral Leadership in Middle Schools in Mainland China
}

\author{
Zhongfeng $\mathrm{Hu}^{\mathbf{1}^{*}}$, Qimei $\mathrm{Li}^{2}$, Shuangxiu Zou ${ }^{3}$ \\ ${ }^{1}$ School of Public Administration, South China Normal University, Guangzhou, China \\ ${ }^{2}$ Shunde Li Zhaoji Middle School, Foshan, China \\ ${ }^{3}$ Zhanjiang Commercial Bank, Zhanjiang, China \\ Email: *Huzhongfe@163.com
}

Received 24 April 2015; accepted 22 May 2015; published 25 May 2015

Copyright (C) 2015 by authors and Scientific Research Publishing Inc.

This work is licensed under the Creative Commons Attribution International License (CC BY).

http://creativecommons.org/licenses/by/4.0/

(c) (i) Open Access

\begin{abstract}
Through literature review, interviews and open questionnaires, we develop a Middle School Principals' Moral Leadership Scale (SPMLS) which suits the Chinese cultural background. Exploratory Factor Analysis ( EFA) of a sample of 92 valid questionnaires shows that the moral leadership of middle school principals takes on a clear five-dimensioned model with factors named Fairness and Justice, Interpersonal Care, Integrity and Commitment, Virtue Cultivation, and Reflective Practice. To further verify the reliability and the validity of this scale, Confirmatory Factor Analysis (CFA) is carried out on another sample of 376 valid questionnaires, and the results confirm the rationality of the five-dimensioned model and the good reliability and validity of the scale.
\end{abstract}

\section{Keywords}

Middle School Principal, Moral Leadership, Structure

\section{Introduction and Literature Review}

\subsection{An Overview of Studies on the Structure of Principal's Moral Leadership in Middle School}

A thorough review of the concerned literature shows that in the past leadership theories mostly focused on leaders' traits, behaviors or techniques, but the aspects of moral cultivation and beliefs of values were often over-

${ }^{*}$ Corresponding author. 
looked or ignored. Production and efficiency tended to be the main pursuits of a leader; nevertheless, there existed other viewpoints, claiming it was a leader's responsibility to ensure ethical standards and moral behaviors. School Moral Leadership theory was first postulated by Sergiovanni (Sergiovanni, 1992), who conducted a rich literature review of predecessors' researches, in his book Moral Leadership: Getting to the Heart of School Improvement. According to him, leadership is more of an attitude than the application of techniques; moral leadership, supposed to be in the central position of a leadership, could be understood as school leaders applying all kinds of feasible methods, in accordance with moral authority, to stimulate the potentiality of people, to make subordinates his followers, and to jointly establish a virtuous school leadership. Following the same line, this study holds the view that the principal's moral leadership refers to the principal guiding school staff to follow his moral charm imperceptibly, on the basis of his moral authority, so that naturally correct ethics and values are established, the staff's sense of obligation and responsibility is inspired, and further triggered are their consciousness to complete tasks, their dedication to school development, and their joint efforts for benign operation and a school development vision.

In recent years, with the research on moral leadership theory conducted more comprehensively and in depth, the related empirical studies have been launched. Scholars put forward different opinions about the structure of moral leadership, with representative views as follows.

Resick, C. J., et al. (Resick et al., 2006) determined the main characteristics of a moral leader on the basis of a review of the concerned western literature on leadership and moral. With the data obtained from the Global Leadership and Organizational Behavior Effectiveness (GLOBE) project and through analysis, they proposed four dimensions of moral leadership, namely Integrity, Altruism, Collective Motivation, and Incentive, which play an important role in the effectiveness of leadership under cross-cultural background.

De Hoogh and Den Dartog (Hoogh \& Dartog, 2008) took the similar understanding of moral leadership as Brown and others but held a different view on the scale of moral leadership, suggesting that it should be a three-dimensioned format with Morality and Justice, Role Clarification, and Power Sharing, the three of which are further composed of their sub-dimensions of 6,5 , and 6, respectively. All of the items of this scale originated from a multi-cultural leadership behaviour questionnaire (MCLQ).

Ladkin (Ladkin, 2008) believed that "good moral leadership" has three main dimensions: Master-understanding of self and the environment, Coordination-all forms of self, purpose and consistency in terms of information, and Purpose - a certain goal achieved. He considered that "good moral leadership" should play a role in "the moral aspects of a leader's efforts" and question whether guided by the purpose of "working hard for people's best interests of living conditions".

Besides, research on principal moral leadership is widespread since the mid of 1990s in Taiwan. Yan Tongwen (Yan, 2002) from National Chi Nan University of Taiwan summarized, in his master's thesis, about the connotation of moral leadership, which includes six dimensions: 1) that the implementation of moral leadership is the most specific performance of the post-modern period; 2) that the spirit of moral leadership focuses on unique charms of individual leaders; 3) that the enforcement of moral leadership is established on the basis of moral authority; 4) that the purpose of moral leadership is to cultivate members' sense of obligation guided by values; 5) that the role of moral leadership is to be proactive but not to retreat; 6) that the dream of moral leadership is to build a virtue school for children.

\subsection{Reflection to Structural Studies on Middle School Principals' Moral Leadership}

In recent years, moral leadership study has achieved fruitful results in literature (a lot of them adopting the research methods of literature study and logic analysis), but in the field of empirical research, there has not yet been enough attention from academic circles.

Although documents of moral leadership are in surge, most of them concentrate on the executive leadership and studies on "moral leadership concepts which tend to be vague and simple". And some of these researches focus on understanding moral leadership theories in the civil administrations (Ciulla, 1998). Other scholars focus on the moral development of leaders and followers. Still others explore the relationship between moral leadership and organizational environment and its influence on employees' satisfaction, performance, commitment, or citizenship behaviors (Trevino et al., 2000, 2003).

Overall, moral leadership has not drawn adequate attention from researchers. Scholars' discussions of moral leadership mainly focus on the theories and concepts of this term, while empirical studies of it are still very 
few.

Brown with his team carried out an empirical study of moral leadership in 2003. They interviewed 20 managers and 20 administrators, developed their Ethical Leadership Scale (ELS), and conducted a field investigation, testing and verification of the structure of organizational moral leadership (Brown et al., 2002; 2005). In addition, studies on moral leadership in school domain mainly focused on the school level (Han, 2006; Liao, 2008), but few have yet taken the core leader of a school—the principal—as the object of moral leadership research.

\subsection{Research Purpose}

This study aims to achieve the following purposes:

1) With a comprehensive synthesis of existing research results and on the basis of a sample investigation adopting interviews and questionnaires, developed is a middle school principal moral leadership scale.

2) A middle school principal moral leadership structure model is to be built, by way of applying exploratory factor analysis, followed by the verification of the model through confirmatory factor analysis.

\section{The Study}

\subsection{Sample}

This study selected teachers from 4 middle schools in Guangzhou, Shenzhen, and Zhanjiang as research subjects. In the pilot tests, 100 questionnaires were distributed, of which there are 92 valid after the invalid being got rid of from the 96 collected back. The effective recovery rate is $92 \%$. And the subjects include 38 male teachers (the effective percentage of $41.3 \%$ ) and 54 females (the effective percentage of $58.7 \%$ ). The formal survey is distributed to 400 subjects, again with the invalid questionnaires taken out, with 376 valid ones recollected, accounting for the $96.2 \%$ of the effective percentage. In detail, among the valid, 203 are male subjects (with an effective percentage of 54\%), and 173 females (with an effective percentage of 46\%).

\subsection{Instruments}

This study adopted a self-made scale, which is developed as follows. Firstly, we conducted a comprehensive search for relevant literature at home and abroad, and analyzed existing items and structure of moral leadership of school principals and corporate leaders, with reference to connotation and existing results of the structure or dimensions of moral leadership of current studies. Secondly, we conducted an investigation of 10 middle school teachers, with open questions, taking as an example of the question of the topic of "what aspects do you think can reflect principals' moral leadership". The teachers' responses helped to get an understanding of teachers' standards of evaluating middle school principals' moral leadership. Then through key word analysis and with proper comprehension and generalization, items to describe related behaviors of middle school principals' moral leadership were determined. Thirdly, we invited teachers to re-arrange the developed items according to the degree of importance (with 5 to 1 to mean from highly important to least important), and to add those items which are not yet included in the list but equally important as the ones already there. Fourthly, adopting the method of critical incident technique (CIT), we got a random sample of 30 middle school teachers from a provincial school and a municipal school in Guangzhou city, and conducted the after-work interviews with them. The interview question was "What aspects do you think can reflect principals' moral leadership? Please give detailed examples.” Then again, through key word analysis, items about middle school principals' moral leadership behavior were singled out and then were compared with those items obtained from the former first two steps. Fifthly, with the data from the interviews and open questionnaires classified, sorted and analyzed, and on the basis of consulting with the subject teachers, all the items were assessed with respect to readability, content relevance, clarity, and construct appropriateness before the formation of our initial inventory of 42 items. Then conducting the item analysis, factor analysis and reliability analysis, we developed the final version of the Middle School Principals' Moral Leadership Scale (SPMLS), which contains 30 items, consisting of 21 positive items and 9 reverse ones. Originally there were 44 items with 2 for lie detection, deletion of which would lead to a total of 42 .

\subsection{Procedure and Statistical Method}

On the basis of a comprehensive review of domestic and foreign literature and the results of open questionnaires 
and interviews, a scale was made with the generalized and universal items determined in terms of middle school principals' moral leadership. Then a pilot study was conducted with the very scale, in which participants were required to tick a degree of judgement which suited their actual situation for each item. After factor analysis, the final SPMLS was formed, and the formal survey was administered to find out the final version of dimensions of the principal's moral leadership. With all questionnaires collected back, confirmatory factor analysis was conducted on the scale to verify the fitting degree of the model as well as reliability and validity of the scale. Statistical work of this study was supported by SPSS 11.0.

\section{Results}

\subsection{Item Analysis}

Through item analysis we obtained item inspection performance indicators, with which we selected proper items so as to improve the validity of the scale. Based on the related theory of measuring, we arranged the pilot test questionnaires of SPMLS from high to low according to the total scores, and then divided them into a highscored group and a low-scored one, with each group having $27 \%$ of the total subjects; followed is the calculation of the discrimination indexes of each item (see Table 1).

According to the item discrimination indicators shown in Table 1 , the T values of items numbered 4, 7, 8, 11, 30 were not statistically significant and therefore should be deleted; thus, there were 37 items left in the scale.

Table 1. Item analysis of the school principal's moral leadership ( $\mathrm{N}=92)$.

\begin{tabular}{|c|c|c|c|c|c|}
\hline Item & Determination Value & Note & Item & Determination Value & Note \\
\hline 1 & $9.830^{* * *}$ & & 23 & $13.824 * * *$ & \\
\hline 2 & $12.793^{* * *}$ & & 24 & $5.526 * * *$ & \\
\hline 3 & $8.963^{* * *}$ & & 25 & $3.957 * * *$ & \\
\hline 4 & .528 & Deleted & 26 & $9.937 * * *$ & \\
\hline 5 & $8.173^{* * *}$ & & 27 & $15.298 * * *$ & \\
\hline 6 & $8.863^{* * *}$ & & 28 & $15.042^{* * *}$ & \\
\hline 7 & -.947 & Deleted & 29 & $9.697 * * *$ & \\
\hline 8 & 1.060 & Deleted & 30 & 1.206 & Deleted \\
\hline 9 & $8.915^{* * *}$ & & 31 & $13.180^{* * *}$ & \\
\hline 10 & $13.540 * * *$ & & 32 & $14.239 * * *$ & \\
\hline 11 & 1.892 & Deleted & 33 & $12.604 * * *$ & \\
\hline 12 & $12.045^{* * *}$ & & 34 & $11.911^{* * *}$ & \\
\hline 13 & $20.209 * * *$ & & 35 & $17.180^{* * *}$ & \\
\hline 14 & $21.453^{* * *}$ & & 37 & $10.340^{* * *}$ & \\
\hline 15 & $13.270 * * *$ & & 38 & $13.884 * * *$ & \\
\hline 16 & $7.045^{* * *}$ & & 39 & $15.963^{* * *}$ & \\
\hline 17 & $14.904^{* * *}$ & & 40 & $12.153^{* * *}$ & \\
\hline 19 & $9.252 * * *$ & & 41 & $10.098 * * *$ & \\
\hline 20 & $12.033^{* * *}$ & & 42 & $10.336^{* * *}$ & \\
\hline 21 & $5.221 * * *$ & & 43 & $7.210^{* * *}$ & \\
\hline 22 & $15.345 * * *$ & & 44 & $13.732 * * *$ & \\
\hline
\end{tabular}

Note: ${ }^{*} p<0.05,{ }^{* *} p<0.01,{ }^{* * *} p<0.001$. 
From Table 1, it could also be seen that all these 37 items have very good discriminability and can identify the reaction degree of different subjects.

\subsection{Exploratory Factor Analysis}

Exploratory factor analysis (EFA) was conducted of the 92 valid questionnaires in the pilot test and the results show that the sample KMO value is 0.932, and the value of Chi-squire in Bartlett spherical is 3853.741, $p<$ 0.001 , statistically indicating very significant level, showing that the data is suitable for factor analysis.

In conducting EFA we first applied main component analysis to extract factors, then used orthogonal varimax method for factor rotation, and next selected factors whose eigenvalue was greater than 1 . Thus with reference to Scree Plot, factors or items are determined. We adopted the same principle of deletion as in the pilot test for item analysis, which helped remove 7 items with the rest of 30 others remaining, composed of 21 positive ones and 9 reverse ones. Main component analysis, with orthogonal varimax rotation, was conducted on the 30 items. Results show that the middle school principal's moral leadership is clearly characterized as a five-dimensioned structure, with factor loadings of all items in the corresponding dimension ranging from 0.417 to 0.833 and the explained variance rate being77.467\%.

With a thorough understanding of the content of the five factors standing for, we used the following concepts to name them. Factor 1 is named as Fairness and Justice, which includes 6 items concerning whether principals handle school affairs with professional perspectives of leadership and the universal principle of moral action. Factor 2 is named as Interpersonal Caring, which has a total of 7 items, all concerning whether principals show generosity and tolerance in teachers' affairs, take the initiative to care for teachers, and promote professional development of teachers. Factor 3 is named as Integrity and Commitment, the 6 items for which involve whether principals keep promises, take responsibilities, attach importance to communication, or care about school development in school management. Factor 4 is named as Virtue Cultivation, which has 6 items involving principals' cultivation of morals, personality traits, and interpersonal sincerity. Factor 5 is named as Reflective Practice, whose 5 items are mainly concerned with principals' self-reflection and willingness to accept suggestions in the course of management, and courage to carry out reforms and to work hard for bringing about the innovation of the school. The above analysis and results are merely conducted and obtained through statistical analysis. As for whether the model is sound and effective, confirmatory factor analysis needs to be conducted for the verification.

\subsection{Confirmatory Factor Analysis}

A second time of factor analysis was conducted on the formal SPMLS, with the following two purposes. Firstly, it aims to re-verify the validity of the pilot-test scale, and secondly, to find out the structured dimensions of middle school principals' moral leadership.

KMO measure and Bartlett spherical test were done as first steps. The KMO value is 0.931 , meaning that the scale is suitable for factor analysis. The Bartlett value is 12404.41, with the statistical significance level less than 0.001 , which further confirms the appropriateness of factor analysis on this scale.

Orthogonal rotation was also done to the formal data through principal component analysis (PCA) to extract factors whose characteristic root is greater than 1 . Similar to the pilot test results, five factors are extracted and the cumulative variable is $76.670 \%$. All factor loadings are above 0.40 . And all factor item results are roughly the same as in the pilot, only with some difference on the amount, which shows that the scale has good construct validity. Thus, not only the results of the pilot test are confirmed, but also proved are the stability of the measurement effect.

\subsection{Reliability Test}

As we know, internal consistency reliability is often indicated by a Cronbach $\alpha$ coefficient. According to DeVellis (1991), a reliability value of 0.70 or above is acceptable; and a value of 0.60 or even below means that the research instrument needs to be revised or even re-written. Therefore, a general reliability coefficient of 0.70 or above can be accepted in respect to the requirements of psychometrics. Upon examination, the self-made SPLMS in this research has good reliability and validity. Internal consistency reliability of all subscales is respectively $0.907,0.893,0.891,0.919$ and 0.921 , and the whole scale's is 0.978 , indicating that the evaluation 
questionnaire of SPMLS has good reliability and stability, and the research results are trustworthy and reliable.

\subsection{Validity Test}

\subsubsection{The Construct Validity}

Construct validity indicates the extent to which a psychological test precisely verifies the theoretical construct of a prepared test. The results from both the exploratory factor analysis and confirmatory factor analysis show that the SPMLS has a clear structure, with all indicators fully meeting the requirements of psychometrics. At the same time, the results of confirmatory factor analysis are in consistency with the theoretical construct initially put forward by the researchers of this study. In fact, observation of the correlation between every item in each dimension and the total score of each corresponding dimension confirms the construct validity of the scale.

As shown in Table 2, the correlation coefficients of each dimension vary between 0.30 and 0.50 , which fall within the range required by psychological scaling method, according to which the correlation between each dimension should be medium or low, ranging from 0.10 to 0.60 , which suggests that each dimension has adequate independence within the scale. The correlation between the total scale and each dimension ranges from 0.60 to 0.90 , showing high correlation with the significance level of 0.001 , also statistically indicating that all 5 dimensions are high in overall consistency. To sum up, the results show that the SPMLS has good construct validity.

\subsubsection{The Content Validity}

Content validity refers to the appropriateness and representativeness of the contents or items of a scale, which means that the test content should be able to reflect the targeted psychological traits that are aimed to be tested. The contents of the SPMLS were originally compiled on the basis of studies of existing literature concerning moral leadership, and on the analysis of the data obtained through investigation of teachers of different genders, ages, subjects and professional titles. In the process of developing the scale, the researchers constantly consulted school administers and common teachers in ordinary secondary school as well as education management experts from universities for judgement and approval of dimension contents, asking them to assess and to make comments on the dimensions of the contents. With the developed scale, the 92 subjects are studied and research results show that the content of these dimensions can basically reflect the content structure of principals' moral leadership in Chinese middle schools, and that the SPMLS has good content validity.

\section{Analysis and Discussion}

As an instrument of this study, developed and adopted is the Middle School Principals' Moral Leadership Scale (SPMLS), which consists of 5 dimensions, namely, Fairness and Justice, Interpersonal Caring, Integrity and Commitment, Virtue Cultivation, and Reflective Practice. Compared with the dimensional structures proposed by western scholars, this one is different from them in many aspects, although there are also some similarities. To be exact, the western understanding of moral leadership puts more emphasis on the role and effect of fairness, integrity, personal beliefs, values, and self-motivated practice of leaders, while less on interpersonal caring. Related studies of moral leadership in mainland China have shown much concern for fairness, integrity and reflective practice; in addition, interpersonal caring and virtue cultivation have also received special attention. The related researches of moral leadership from Hong Kong and Taiwan have not only covered fairness and justice,

Table 2. Correlation between items and total scores of corresponding dimensions in the SPMLS $(\mathrm{N}=376)$.

\begin{tabular}{|c|c|c|c|c|c|c|}
\hline & Factor 1 & Factor 2 & Factor 3 & Factor 4 & Factor 5 & Factor 6 \\
\hline Factor 1 & 1 & & & & & \\
\hline Factor 2 & $.396^{* * *}$ & 1 & & & & \\
\hline Factor 3 & $.417^{* * *}$ & $.326 * * *$ & 1 & & & \\
\hline Factor 4 & $.384^{* * *}$ & $.407 * * *$ & $.374 * * *$ & 1 & & \\
\hline Factor 5 & $.428^{* * *}$ & $.358^{* * *}$ & $.406 * * *$ & $.352 * * *$ & 1 & \\
\hline Factor 6 & $.757^{* * *}$ & $.845^{* * *}$ & $.646^{* * *}$ & $.725^{* * *}$ & $.653^{* * *}$ & 1 \\
\hline
\end{tabular}


democratic participation and reflective thinking, but also focused much on interpersonal caring and virtue cultivation. The existing differences in the research interests may be due to the cultural differences between the East and the West. Compared with westerners worshipping fairness and justice, Chinese Confucian culture values more the role of benevolence and righteousness, morality, and universal love play in the management practice, which has gradually formed the tradition of moral practice in more than two thousand years of governing. It is on the foundation of moral practice that in today's China, at the national level, "ruling the country by virtue" has become the concept of state-governing; while in the field of education, although the moral, intellectual, physical, aesthetic and labour goals are all emphasized in order to develop well-rounded students, the moral education has become the first concern. Thus, under the background of Chinese culture and as an art of school leadership, the principals' moral leadership should not only include traditional concepts such as fairness and justice, integrity and commitment, as well as reflective practice, but also have interpersonal caring and virtue cultivation as its indispensable parts.

\section{References}

Brown, M. E., \& Treviiño, L. K. (2002). Conceptualizing and Measuring Ethical Leadership: Development of an Instrument. Academy of Management Proceedings, 2002, SIM D1-SIM D6. http://dx.doi.org/10.5465/apbpp.2002.7519501

Brown, M. E., Treviiño, L. K., \& Harrison, D. (2005). Ethical Leadership: A Social Learning Perspective for Construct Development and Testing. Organizational Behavior and Human Decision Processes, 97, 117-134. http://dx.doi.org/10.1016/j.obhdp.2005.03.002

Ciulla, J. B. (1998) Ethics, The Heart of Leadership. Westport: Greenwood, P.G78-90.

De Hoogh, A. H. B., \& Den Dartog, D. N. (2008). Ethical and Despotic Leadership, Relationships with Leader's Social Responsibility, Top Management Team Effectiveness and Subordinates' Optimism: A Multi-Method Study. The Leadership Quarterly, 19, 297-311. http://dx.doi.org/10.1016/j.leaqua.2008.03.002

DeVellis, R. F. (1991). Scale Development: Theory and Applications (Applied Social Research Methods Series, Vol. 26). Newbury Park, CA: Sage Publications.

Han Y. C. (2006). Theoretical Construct and Practice Explore of Moral Leadership of School. East China Normal University, 4, 82.

Ladkin, D. (2008). Leading Beautifully: How Mastery, Congruence and Purpose Create the Aesthetic of Embodied Leadership Practice. The Leadership Quarterly, 19, 31-41. http://dx.doi.org/10.1016/j.leaqua.2007.12.003

Liao, X. (2008). An Overview of Researches into Moral Leadership of School. Journal of Mudanjiang College of Education, 4, 63.

Resick, C. J., Hanges, P. J., Dickson, M. W., \& Mitchelson, J. K. (2006). A Cross-Cultural Examination of the Endorsement of Ethical Leadership. Journal of Business Ethics, 63, 345-359. http://dx.doi.org/10.1007/s10551-005-3242-1

Sergiovanni, T. J. (1992). Moral Leadership: Getting to the Heart of School Improvement. San Francisco: Jossey Bass Publish.

Trevino, L. K., Brown, M., \& Hartman, L. P. (2003). A Qualitative Investigation of Perceived Executive Ethical Leadership: Perceptions from Inside and Outside the Executive Suite. Human Relations, 55, 5-37. http://dx.doi.org/10.1177/0018726703056001448

Trevino, L.K., Hartman, L., \& Brown, M. (2000). Moral Person and Moral Manager: How Executives Develop a Reputation for Ethical Leadership. California Management Review, 4, 128.

Yan, T. W. (2002). The Study on Primary School Principal's Moral Leadership in Four Counties of Middle Taiwan. Chi Nan University of Taiwan, Nantou, Taiwan. 